\title{
Herzlich willkommen, FMH!
}

\author{
Ursina Pally Hofmann
}

Dr. iur., Generalsekretärin der FMH und Leiterin Abteilung Rechtsdienst

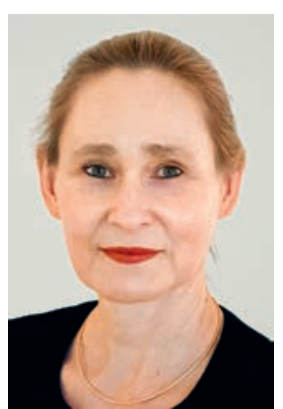

Als ich am 1. Juni 2018 mit diesem Gedanken meine Tätigkeit als Leiterin Rechtsdienst bei der FMH aufgenommen habe, wusste ich bereits, dass mich ein äusserst interessanter Job erwarten würde. Für eine Hebamme und Rechtsanwältin ist es heute spannender denn je, an einem der Hotspots des Schweizer Gesundheitswesens tätig zu sein.

Um welche Dimensionen sich meine Aufgabe bei der FMH in den folgenden Monaten erweitern sollten, konnte ich damals noch nicht ahnen. Als Generalsekretärin und damit operative Leiterin des «KMU-Betriebs» FMH kümmere ich mich seit dem

1. Oktober 2018 zusätzlich unter anderem um Personal-, Finanz- und IT-Themen, begleite Projekte und trage die Verantwortung für den reibungslosen Ablauf der verbandsinternen Sitzungen. Die Zusammenarbeit mit dem Präsidenten, dem Zentralvorstand und weiteren Organen sowie den Ärztegesellschaften und Dachorganisationen bringt es mit sich, dass ich regelmässig auch in die politischen Themen einbezogen werde.

Eine konstruktive und wertschätzende Zusammenarbeit wird es uns erlauben, auch anspruchsvolle Aufgaben zu meistern.

Die Herausforderungen des «KMUs» FMH sind vielfältig und gross, nachfolgend nenne ich nur ein paar wenige. Die FMH-Liegenschaft in der Berner Elfenstrasse ist sanierungsbedürftig. Seit mehr als einem Jahr sind wir daran, diese Erneuerung im Rahmen des Ärztekammerbeschlusses umzusetzen. Ein weiteres Projekt zielt darauf ab, unsere Arbeitsweise mittels eines modernen Rekord-Management-Systems effizienter und sicherer zu gestalten. Die digitalen Schnittstellen zu den angeschlossenen Gesellschaften sind veraltet, und die bereits geltenden, aber auch die noch folgenden Änderungen in der Datenschutzgesetzgebung erfordern Anpassungen bei der IT und im täglichen Umgang mit dieser. Der Service «Gutachterstelle» soll neuen Bedürfnissen gerecht werden, weshalb ein neues Verfahrensreglement im Entstehen ist. Weiter ist unser Rechtsleitfaden für den medizinischen Alltag nicht mehr auf dem neuesten Stand und ebenfalls in Überarbeitung. Ein Dauerbrenner sind die Tarifverhandlungen, welche unsere Kolleginnen und Kollegen in Olten seit Jahren beschäftigen. Die Präsenz von

Ich kann auf ein tolles Team zählen: Erfahrene Mitarbeitende mit viel FMH-Know-how sowie neue Mitarbeitende mit neuen Ideen.

Gesundheitsthemen in den Medien führt dazu, dass auch die Abteilung Kommunikation überaus gefordert ist. Alleine im Monat Oktober sind 60 Medienanfragen eingegangen. Diese Beispiele geben Ihnen einen kleinen Einblick in unser tägliches Wirken im Generalsekretariat.

Neben mir haben dieses Jahr weitere Kolleginnen und Kollegen ihre Tätigkeit bei der FMH aufgenommen. Erfreulicherweise kann ich mich auf ein sowohl fachlich als auch menschlich sehr gutes Team verlassen. Einerseits verfügen langjährige und erfahrene Kolleginnen und Kollegen über das relevante FMHKnow-how, andererseits bringen die neu dazugekommenen Mitarbeitenden neue Ideen, frischen Wind und ihre Erfahrungen aus anderen Bereichen oder Branchen mit ein. Ich freue mich auf eine konstruktive und wertschätzende Zusammenarbeit, die es uns erlauben wird, auch anspruchsvolle Aufgaben zu meistern.

Nicht minder freue ich mich darauf, weitere Bekanntschaften unter Ihnen - den Mitgliedern der FMH zu machen und mit Ihnen zusammenzuarbeiten. Wir dürfen wohl davon ausgehen, dass es im Gesundheitswesen spannend bleibt. Lesen Sie in dieser Ausgabe auf Seite 1825 gleich selbst, was es beispielsweise mit dem neuen Verjährungsrecht auf sich hat und was dieses für Sie ganz persönlich bedeuten könnte. 